Patrice PINELL, La Bonne Société et la cause de la petite enfance. Sociogenèse de la première loi française de protection de l'enfance (1874)

\title{
François Buton
}

\section{(2) OpenEdition}

Journals

Édition électronique

URL : http://journals.openedition.org/ress/6672

DOI : $10.4000 /$ ress.6672

ISBN : 1663-4446

ISSN : $1663-4446$

Éditeur

Librairie Droz

Édition imprimée

Date de publication : 1 décembre 2020

Pagination : 268-272

ISSN : 0048-8046

Référence électronique

François Buton, «Patrice PINELL, La Bonne Société et la cause de la petite enfance. Sociogenèse de la première loi française de protection de l'enfance (1874) 》, Revue européenne des sciences sociales [En ligne], 58-2 | 2020, mis en ligne le 01 décembre 2020, consulté le 03 décembre 2020. URL : http:// journals.openedition.org/ress/6672; DOI : https://doi.org/10.4000/ress.6672

Ce document a été généré automatiquement le 3 décembre 2020.

(c) Librairie Droz 


\title{
Patrice PINELL, La Bonne Société et la cause de la petite enfance. Sociogenèse de la première loi française de protection de l'enfance (1874)
}

\author{
François Buton
}

\section{RÉFÉRENCE}

Patrice PINELL, 2019, La Bonne Société et la cause de la petite enfance. Sociogenèse de la première loi française de protection de l'enfance (1874), Vulaines-sur-Seine, Éditions du croquant, $342 \mathrm{p}$.

1 Auteur d'ouvrages classiques sur la naissance du cancer et du sida, mais aussi de plusieurs articles majeurs sur l'histoire du champ médical de la Révolution au deuxième $\mathrm{XX}^{\mathrm{e}}$ siècle, le sociologue Patrice Pinell propose ici une analyse fouillée des conditions qui ont rendu possible, dans les années 1860 et 1870, une rupture profonde dans la manière de penser la mort des nourrissons et ont, à plusieurs égards, concouru à faire de la protection de la petite enfance une politique publique préfigurant l'État social en France. L'analyse montre ce que la préparation et l'élaboration de la fameuse loi Roussel instituant la protection de l'enfance en 1874 doit à l'action conjuguée, et souvent conjointe, d'une société philanthropique - la Société protectrice de l'enfance (SPE) - et de différentes fractions de la profession médicale.

2 Travaillant depuis longtemps sur les processus de construction des spécialités médicales, notamment de la pédiatrie, Pinell ouvre une perspective nouvelle sur un sujet déjà travaillé par l'historienne Catherine Rollet. Il le fait en sociologue spécialiste du champ médical, qui récuse l'idée générale d'une " prise de conscience » du problème de la mortalité infantile par les savants, et d'abord par les médecins, lesquels parviendraient ensuite à l'imposer aux autorités publiques (une sorte de " réalisation 
du rêve hygiéniste »), et qui se donne pour tâche de reconstruire l'espace des luttes pour la définition du problème afin de comprendre depuis quelle position sociale, et selon quels intérêts propres, sociopolitiques, idéologiques et politique, certains acteurs imposent une nouvelle approche de la mortalité infantile. On rappellera qu'avec un taux qui s'élève à $20 \%$, cette dernière constitue encore à l'époque une expérience " partagée ", radicalement éloignée de la nôtre. Contre la perspective simplificatrice de la prise de conscience, qui est aussi la version officielle des législateurs, à commencer par le député Théophile Roussel, auteur du projet de loi en 1873, Pinell reconstitue méticuleusement le milieu réformateur dans lequel, à côté des médecins lanceurs d'alerte, des membres de l'Académie de médecine et de ceux des commissions officielles, les philanthropes figurent au premier rang. Or la réintégration dans l'analyse des acteurs de la bienfaisance constitue un pas décisif non seulement en ce qu'elle rend mieux compte du tableau complexe de la réalité historique d'alors, mais aussi en ce qu'elle ouvre une réflexion sur le rôle de la classe bourgeoise, que Pinell conceptualise comme la «Bonne Société » (en italique dans le titre de l'ouvrage), c'està-dire l'ensemble des acteurs qui se perçoivent eux-mêmes et entre eux comme détenteur d'un statut social qui les distingue (et les sépare) des membres des classes populaires - autrement dit, qui détiennent non seulement des capitaux économiques et culturels, mais accumulent un capital symbolique les autorisant à s'engager dans le champ de la bienfaisance pour encadrer à leur tour les conduites des pauvres au nom d'une vision binaire du monde social. Ce champ de la bienfaisance, depuis longtemps structuré selon la double opposition entre associations de statut privé et institutions de statut public d'une part, et entre œuvres catholiques et laïques d'autre part, obéit aussi à un principe de hiérarchie qui place la "bonne société » parisienne bien au-dessus des "bonnes sociétés" des villes de province. La question au cœur de l'entreprise philanthropique est bien sûr la construction d'une catégorie sociale nouvelle, les nourrissons, dont le sort ne peut plus être réservé à leurs familles, mais doit impliquer l'initiative philanthropique et l'action étatique. Cependant, elle réside aussi et surtout dans le contrôle par l'État d'une activité répandue et sujette à critiques, la mise en nourrice des nouveau-nés, qui met en question à la fois l'autorité paternelle, la place de l'allaitement maternel, et le rôle imposé aux femmes, donc la domination masculine.

3 L'analyse sociogénétique se déploie très classiquement en trois parties qui se succèdent chronologiquement: d'abord la constitution jusqu'en 1865 d'une nouvelle cause, la surmortalité infantile; ensuite la traduction de cette cause en problème politique, dans la seconde moitié des années 1860 ; enfin, après la guerre de 1870, l'élaboration et le vote de la loi et les débuts de sa mise en œuvre.

4 La première partie présente en deux chapitres comment la surmortalité des enfants placés en nourrice est construite comme un problème social majeur. Le premier chapitre revient sur les conditions du changement de regard sur la mortalité infantile. La prise en charge des enfants en bas âge "trouvés ", et placés en nourrice par un bureau qui dépend de l'Assistance publique à partir de 1849, intéressent peu les médecins des hôpitaux et les philanthropes : la clinique des enfants malades s'intéresse à la description des symptômes et au classement des entités morbides plutôt qu'à leur étiologie, cependant que les philanthropes se préoccupent surtout du placement en " salle d'asile » puis en "crèche » des enfants de plus de deux ans des ouvrières. Les médecins de famille, en revanche, défendent l'allaitement maternel auprès des mères des milieux aisés qui constituent leur clientèle, et débattent de l'alternative offerte par la mise en nourrice (ou par l'alimentation par le lait animal), qu'ils entendent encadrer, 
en participant au recrutement des nourrices (évaluation des qualités physiques et morales) et en leur imposant des règles (interdiction d'allaiter leur propre enfant et de tomber enceinte). L'importance de la mortalité des enfants trouvés et placés en nourrice est mise à jour dès les années 1820 , mais c'est le développement d'une « industrie des nourrices » par des bureaux privés qui, au tournant des années 1840, fait problème. Cette industrie met en question les pratiques du Bureau des nourrices de l'Assistance publique et vient capter la clientèle des médecins de famille; elle fait dès lors du placement des nourrissons un enjeu entre les tenants d'un contrôle par l'État et ceux d'une régulation par le marché. Le deuxième chapitre porte sur la genèse sous influence fouriériste de la SPE, en 1865, et présente une analyse méticuleuse de deux ouvrages décisifs, critiques à l'égard de l'industrie des nourrices, rédigés respectivement par les docteurs Monot et Brochard. On dispose ainsi d'éléments probants sur les promoteurs, au sein de la SPE, d'une remise en cause du placement des nourrissons, perçue comme un désengagement préjudiciable des familles dans l'élevage des enfants, et sur les désaccords qui opposent l'un de ses fondateurs, le chirurgien lyonnais François Barrier, militant fouriériste partisan de colonies maternelles conçues sur le modèle du phalanstère, et les patrons parisiens de l'élite médicale qui rejoignent la SPE à partir de 1866 et défendent la mise sur pied d'un réseau de médecins volontaires pour surveiller les enfants au domicile des nourrices. On comprend aussi les logiques qui président aux critiques de Monot et Brochard et les amènent à plaider en faveur d'une intervention de l'État, entre dénonciation morale des abus des bureaux privés et des nourrices, et mise en évidence empirique, par des données quantitatives, de la surmortalité relative des enfants qui y sont placés.

5 La deuxième partie, également composée de deux chapitres, porte respectivement sur les débats menés à l'Académie de médecine et sur la réorientation stratégique de la SPE en faveur de la surveillance médicale des nourrices. Le troisième chapitre détaille ainsi les enjeux scientifiques, idéologiques et politiques de la discussion menée tout au long des 27 séances au sein de l'institution savante : ils concernent d'une part l'analyse du problème de la mortalité infantile et d'autre part la définition du rôle de l'Académie et la nature du travail pour accomplir sa mission. Les tenants d'un libéralisme plus ou moins radical s'opposent en effet aux partisans d'une réglementation éclairée par la science. Une enquête établit une surmortalité considérable des nourrissons placés en nourrice par rapport aux nourrissons allaités par leur mère et un large consensus s'établit pour faire de "l'immoralité » la cause première de la mortalité infantile dans les classes pauvres. Pourtant, l'Académie, tiraillée par des oppositions internes, s'interdit de prendre position, préférant dégager l'éventail des mesures susceptibles de remédier au problème plutôt que d'en retenir une, et laisser aux autorités politiques la responsabilité de la décision. Ensuite, le quatrième chapitre examine la fin de l'influence fouriériste au profit d'un intense travail de propagande en faveur de l'allaitement maternel et de la surveillance des nourrissons placés par des médecins volontaires. Pinell souligne notamment comment des médecins de campagne (au nombre de 350 en 1870) accumulent, par leur action bénévole et bienfaisante dans une forme de médecine sociale, du capital symbolique qui leur permet d'accéder à des positions dominantes dans l'élite sociale du monde rural - la notabilisation, dans leur cas, ne résultant ainsi pas seulement de l'exercice de la pratique libérale, contrairement à un mythe répandu.

6 Enfin, la troisième partie étudie la mise en pratique de la politique de protection de l'enfance après la guerre de 1870 , qui se caractérise essentiellement par un partage des 
rôles entre l'État républicain et la philanthropie privée. Le cinquième chapitre répond à une énigme : comment le député Roussel, lui-même médecin et académicien, parvientil à faire voter à l'unanimité un projet de loi sur un sujet aussi débattu? La réponse, grossièrement résumée, réside dans un travail de reprise des positions de la SPE sous une forme à la fois minimale, donc réduite, et décalée. La loi Roussel ne retient pas certaines propositions philanthropiques importantes, comme celle en faveur de l'obligation de l'allaitement maternel, contradictoire avec la toute-puissance et la liberté de choix du chef de famille, ou bien celle en faveur d'une aide spécifique pour les mères ouvrières. Les législateurs s'en tiennent au seul encadrement de l'industrie des nourrices, dont l'État organise le contrôle administratif, médical et social et en délègue le travail de terrain aux notables locaux de la bonne société, dont font partie les membres des SPE (qui prétendaient en avoir le monopole). Le chapitre 6 détaille la division du travail qui se met en place dans l'application de la loi, au moyen d'un dispositif étatique vertical (Comité supérieur, comités départementaux, inspections départementales) qui encadre les agents privés chargés de protéger sur le terrain les enfants placés en nourrices. Il pointe surtout l'écart entre deux types d'agents privés. D'un côté, la surveillance par les dames patronnesses locales et bénévoles rencontre très vite des limites : la délégation de l'autorité de l'État ne suffit pas à rendre légitime l'intervention de bourgeoises et mères de famille face à l'hostilité de classe du petit peuple. De l'autre, celle des médecins est mieux tolérée : or, même s'ils se plaignent de la faiblesse des rétributions, l'activité d'inspection, mission de service public, confère à nombre de médecins libéraux en milieu rural (jusqu'à la moitié d'entre eux dans certains départements) tous les avantages de la médecine sociale publique de type salariée (une rémunération garantie) sans ses défauts (nulle contrainte hiérarchique), et par suite tous les profits symboliques d'un engagement charitable noble donc désintéressé. Autrement dit, les médecins de campagne bénéficient désormais autant financièrement que symboliquement de leur travail de surveillance des nourrices.

7 Comme la conclusion le souligne clairement, la nouvelle politique de protection de la petite enfance soulève ainsi plusieurs questions de société majeures : les modalités de l'exercice de la domination masculine, via le consensus sur la préférence pour l'allaitement maternel, l'accès aux seules pratiques de la bienfaisance dans le champ du pouvoir ou encore les difficultés de l'ingérence étatique dans l'espace privé qui empêche de toucher au statut dominé de l'épouse dans une société patriarcale; la régulation des activités de service public en matière sociale, qui voit la puissance publique agir comme maître d'œuvre - non pas exactement déléguer l'action de terrain mais mobiliser des agents privés, en l'occurrence des médecins libéraux, pour une action non plus bénévole, mais rémunérée - ; les formes d'exercice de la domination de classe, entre rapports contractuels de domesticité (emploi d'une nourrice sur le lieu du domicile), contrôle social par la surveillance médicale (emploi d'une nourrice à la campagne) et assistance aux nécessiteux (octroi d'une aide pour l'allaitement maternel).

On pourra regretter que l'analyse perde parfois un peu de vue la chronologie fine des enchaînements dans le processus de construction à la fois savante, sociale, et politique du problème, notamment en raison du choix thématique des chapitres dans les deux premières parties, ou que les effets de ce processus sur le champ médical, notamment sur les spécialités médicales, ne soient pas davantage systématisés. On regrettera aussi que l'édition de l'ouvrage ne soit pas à la hauteur de sa qualité scientifique, tant au niveau de l'appareillage critique (pas d'index des noms propres, ni de lieux ou 
d'institutions, pas de bibliographie en fin de volume) que de la correction la plus élémentaire (par exemple, le directeur de l'Assistance publique Husson est renommé Huchon p. 156 et suivantes). Mais il faut avant tout saluer un ouvrage dont la démonstration emporte la conviction, et est appelé à faire référence sur le sujet comme sur le fonctionnement du champ du pouvoir de l'époque.

\section{AUTEURS}

\section{FRANÇOIS BUTON}

ENS Lyon, CNRS-Triangle 